\title{
Análisis cuantitativo del consumo tecnológico en infantes y preescolares hispanohablantes
}

Paloma Suárez Brito y Elda Alicia Alva Canto

\section{Resumen}

Los dispositivos tecnológicos que utilizan hoy en día los adultos, se han infiltrado en la vida cotidiana de infantes y preescolares. Sin embargo, estudios recientes han mostrado que el uso excesivo de medios electrónicos ( $>2$ horas al día) en preescolares, genera patrones de sueño alterados, y conductas agresivas, hostiles e hiperactivas.

El propósito del presente estudio fue realizar una evaluación de los hábitos tecnológicos de infantes y preescolares mexicanos entre 1 y 6 años de edad. A través del análisis de 10 variables, obtenidas de la aplicación de un cuestionario via telefónica a los cuidadores de 300 niños(as); se describe el tipo y frecuencia de uso de diferentes dispositivos tecnológicos.

El presente estudio se ofrece como una herramienta para la toma de decisiones informada sobre la pertinencia del uso de dispositivos móviles en edades tempranas.

Palabras clave: consumo tecnológico, tecnología, hábitos tecnológicos, infantes, preescolares 
Hoy en día los dispositivos tecnológicos que utilizan los adultos, son manipulados también por infantes y preescolares en la vida cotidiana (McManis \& Gunnewig, 2012; Yienger, 2016). Los dispositivos móviles se han vuelto rápidamente en los preferidos por los niños, principalmente por sus características físicas como el tamaño de la pantalla, así como la portabilidad, la capacidad para descargar contenido y su cualidad interactiva (Kabali et al., 2015)low-income, minority community. The survey was adapted from Common Sense Media's 2013 nationwide survey. RESULTS Most households had television (97\%. De manera general, se ha descrito que los infantes utilizan los dispositivos móviles para jugar, ver videos, tomar fotografías y acceder a aplicaciones (apps) de distinta índole (Gutnick, Robb, Takeuchi, \& Kotler, 2011; Kabali et al., 2015; Suárez, Perez, Ferreira, \& Alva, 2016)low-income, minority community. The survey was adapted from Common Sense Media's 2013 nationwide survey. RESULTS Most households had television (97\%).

Con el vertiginoso avance de la tecnología, cada vez existen más estudios que analizan cómo dichos dispositivos impactan en la salud y el desarrollo de infantes y escolares. Si bien es cierto que aún no se tiene información concreta de cuáles son los tipos de dispositivos y contenidos específicos que favorecen el desarrollo en los niños, es bien conocido cómo infantes desde edades tempranas interactúan con sus padres utilizando teléfonos inteligentes y tabletas en espacios públicos, como restaurantes y medios de transporte (Slutsky \& DeShetler, 2017). Aunado a esto, se ha descrito en diferentes estudios que aproximadamente el 50\% de los infantes de cuatro años de edad son consumidores tecnológicos múltiples, es decir, que utilizan más de una plataforma digital al mismo tiempo, por ejemplo, televisión y tableta electrónica; y que además utilizan aplicaciones o apps diseñadas para su edad (Gutnick et al., 2011; McClure, Vaala, \& Spiewak Toub, 2017; McDaniel \& Radesky, 2018; Slutsky \& DeShetler, 2017). Algunos estudios han descrito el potencial educacional que las apps tienen sobre el desarrollo y aprendizaje infantil, pero se deben tomar con reserva hasta que no se valide su desarrollo y/o utilización en los niños (Zosh, Hirsh-Pasek, Golinkoff, \& Parish-Morris, 2016). 
En un estudio, Kabali, et al. (2015) describió el consumo tecnológico cotidiano en infantes angloparlantes de 0 a 4 años de edad, en una comunidad urbana de bajos recursos. El autor encontró que la mayoría de los niños tenían acceso a dispositivos móviles en sus casas, y que a la edad de cuatro años tres de cada cuatro infantes ya poseían su propio dispositivo móvil (principalmente tableta electrónica). A la edad de 2 años, la mayoría de los infantes utilizaba los dispositivos diariamente casi en la misma cantidad que la televisión.

Por otro lado, existe literatura que describe cómo el uso de dispositivos tecnológicos puede promover y favorecer habilidades de lenguaje a través de ejercicios prácticos con las letras, sonidos y reconocimiento de palabras (Rees, Nadig, \& Rvachew, 2017; Rvachew, Rees, Carolan, \& Nadig, 2017)specifically comparing children with typically developing language (TD. Específicamente los libros electrónicos o e-books, pueden resultar útiles para promover el desarrollo del vocabulario y comprensión de lectura y pueden ser más llamativos para los infantes debido a las animaciones, los efectos de sonido o los juegos (Rees et al., 2017)specifically comparing children with typically developing language (TD. Sin embargo, el uso de e-books para la promoción del desarrollo de los infantes también se ha descrito como una interferencia en la comprensión de la tarea en turno, ya que esas mismas características (el diseño visual, los efectos de sonido y la característica de pantalla táctil) pueden distraer al infante del contenido educativo dependiendo de su edad (Karemaker et al., 2017; Radesky, Schumacher, \& Zuckerman, 2015). Algunos estudios han mostrado que, desde el punto de vista de los padres, los infantes de 1 a 4 años se involucran más en una actividad de lectura conjunta cuando tienen libros impresos, a diferencia de libros electrónicos (Karemaker et al., 2017; Strouse \& Ganea, 2017). Así mismo, existen informes de la Academia Americana de Pediatría en las que mencionan que no hay evidencia suficiente para apoyar el uso de pantallas táctiles por infantes menores de dos años (Kucirkova \& Zuckerman, 2017; Zosh et al., 2016)teachers, pediatricians and other child health professionals face the challenge of widespread1, increasing2 and multipurpose 3 use of touchscreens. Even toddlers are 
accessing technologies such as tablets, smartphones, iTouch and other mobile devices with increasing frequency. Child use of this engaging technology is associated with diverse beliefs and practices on the part of parents, motivated by what is best for the child and by the difficulty of limiting touchscreen use 4 . The American Academy of Pediatrics (AAP, 20165.

Respecto a los efectos negativos sobre la salud y el desarrollo de los infantes, existen estudios recientes que han mostrado que el uso excesivo de medios electrónicos ( $>2$ horas al día) en preescolares, puede generar patrones de sueño alterados, así como conductas agresivas, hostiles, ansiosas e hiperactivas (Séguin \& Klimek, 2016). Dichos estudios han descrito que a medida que aumenta la edad de los infantes, éstos son más propensos al uso de diferentes dispositivos tecnológicos y por lo tanto, a sufrir algún trastorno clínico o de conducta. En general, la presencia de un televisor en la recámara de los niños está asociada con una disminución de aproximadamente 30 minutos de sueño, en comparación con aquellos niños que no cuentan con una TV en su cuarto. Las dificultades para conciliar el sueño, a su vez pueden avanzar a corto y largo plazo hacia el desarrollo de problemas atencionales y de desempeño académico. En concreto, los niños que acostumbran prácticas que irrumpen en la cantidad o calidad de sueño, tales como el uso de medios electrónicos, puede derivar en problemas cognitivos y de conducta en la edad escolar e incluso en la adolescencia (Radesky et al., 2015; Séguin \& Klimek, 2016).

En una serie de estudios, Suárez y colaboradores (Suárez, Alva, \& Ferreira, 2016; Suárez, Ferreira, \& Alva, 2016; Suárez, Perez, et al., 2016) mostraron que, en infantes hispanohablantes menores a cuatro años de edad, los hábitos tecnológicos son análogos que en infantes de habla inglesa (Gutnick et al., 2011; Suárez, Perez, et al., 2016). La mayor similitud radica en la preferencia de uso del tipo de dispositivo, así como el efecto de la variable edad en la transformación de los hábitos. Este hecho nos indica que, si bien las características demográficas y culturales influyen en el desarrollo, las características conductuales de consumo tecnológico se comparten entre ambas poblaciones, específicamente cuando se trata del nivel socioeconómico medio. 
Los resultados descritos en estudios previos demuestran que el consumo tecnológico comienza cada vez más temprano en el desarrollo, que el uso de dispositivos es frecuente en edades tempranas, que los infantes pueden consumir contenidos en dichos dispositivos de forma independiente y que además existe una propensión al uso simultáneo de dos o más dispositivos. Debido a que el uso de la tecnología se implementa hoy en día en el contexto educativo además del contexto familiar, es imperante conocer los hábitos de consumo para poder utilizar la tecnología en favor de los infantes desde el contexto inmediato, hasta el contexto escolar y social.

Por lo anterior y con el propósito de edificar sobre estudios previos en infantes mexicanos, el objetivo del presente estudio fue realizar una estimación de los hábitos tecnológicos que presentan infantes y preescolares mexicanos entre 1 y 6 años de edad, de nivel socioeconómico medio, residentes de la Ciudad de México.

\section{Método}

\section{Participantes}

En este estudio participaron 325 cuidadores (padres o madres) de infantes entre 12 y 72 meses de edad (155 niños y 170 niñas). La edad promedio de las madres fue de 33.4 (DE=6.4) años y escolaridad promedio de 15.2 (DE=3.2) años de estudio; y la edad promedio de los padres fue de 35 (DE=10.1) años con escolaridad promedio de 14.3 (DE= 4.3) años de estudio.

Así mismo y por convenir a un meta análisis, se agruparon los datos de acuerdo a la edad de los infantes, quedando seis grupos de edad. El primer grupo quedó conformado por 59 infantes de 12 a 23 meses de edad ( $X=19$ meses); el segundo grupo por 62 infantes de 24 a 29 meses de edad ( $X=27$ meses); el tercer grupo por 67 infantes de 30 a 35 meses de edad $(X=33$ meses); el cuarto grupo se conformó por 60 infantes de 36 a 41 meses de edad ( $X=38$ meses); el quinto grupo por 50 infantes de 42 a 47 meses de edad; y un sexto grupo con 27 infantes de 48 a 72 meses de edad. 


\section{Diseño}

El presente estudio es de tipo descriptivo transversal con muestreo probabilístico aleatorio simple (sin reposición), de participación voluntaria.

\section{Instrumentos y materiales}

Se utilizó el Cuestionario de Hábitos Tecnológicos (CHaT) de Suárez, et al. (2016), el cual está basado en el estudio de Gutnick, et al. (2011), sobre hábitos tecnológicos de infantes y escolares estadounidenses, y se conforma de 10 preguntas acerca de los dispositivos que se tienen en casa, así como aquellos que los infantes prefieren utilizar en el día a día.

El tipo de respuesta es dicotómica (sí/no; positivo/negativo), abierta (por ejemplo: ¿Cuánto tiempo pasa el infante con cada dispositivo?), y de opción múltiple considerando 10 opciones, correspondientes a nueve tipos de dispositivos diferentes, más el acceso a internet como décima opción. Los dispositivos tecnológicos considerados en el cuestionario son: teléfono inteligente, teléfono celular no inteligente, computadora portátil, PC de escritorio, reproductor $\mathrm{mp3}$, videojuego portátil, consola de videojuego, tableta electrónica y televisión.

Los cuidadores podían dar más de una respuesta en las preguntas, a excepción de aquellas en las que el tipo de respuesta es dicotómica o solicita información sobre el tiempo (número de minutos) que el infante pasa con los dispositivos. En el caso de este último tipo de pregunta, las respuestas se agrupan en rangos de tiempo en las opciones de: 0 min, 1-15 min, menos de una hora, 1-2 horas, 3-5 horas y más de 5 horas. Dos de las diez preguntas además, se refieren al tiempo que los infantes destinan a actividades de juego y lectura, sin considerar el uso de dispositivos tecnológicos. 
Las preguntas que conformaron el cuestionario se presentan a continuación:

1. ¿Qué dispositivos tiene en casa?

2. ¿Cuál es el medio favorito del infante?

3. ¿Cuánto tiempo pasa el infante con cada dispositivo en un día normal?

4. ¿El infante tiene dispositivo propio?

5. ¿Qué contenidos ve el infante?

6. ¿En qué contexto usa el infante los dispositivos?

7. Personas con quien el infante usa el dispositivo.

8. Tiempo aproximado que ocupa el infante en JUEGO al día (sin considerar el uso de dispositivos):

9. Tiempo aproximado que ocupa el infante en LECTURA al día (sin considerar el uso de dispositivos):

10. ¿Cómo considera el uso de dispositivos con su bebé?

\section{Procedimiento}

Se realizó la selección de los participantes de una base de datos de acceso restringido, creada y utilizada con fines de investigación con infantes por la institución en la que colaboran los autores de este trabajo. Dicha base contiene información básica (edad, género, datos de contacto, problemas perinatales) de infantes de entre 6 y 84 meses de edad, cuyos padres brindaron sus datos de manera voluntaria y accedieron a ser contactados para participar en el estudio. Los criterios de selección de los participantes fueron que tuvieran entre 12 y 72 meses de edad y que no hubieran presentado problemas antes, durante y después del parto (información que podía ser consultada en la misma base de datos).

Una vez identificados a los participantes en la base de datos, se contactó vía telefónica de manera individual a cada uno de los 325 cuidadores participantes que conformaron la muestra de esta investigación. Después de que el cuidador reiterara su aprobación de participar voluntariamente en el estudio y una vez que se garantizó verbalmente la confidencialidad de sus respuestas, se procedió a aplicar el cuestionario ChaT. Todas las llamadas telefónicas y por tanto la aplicación del cuestionario 
fueron realizadas por cuatro investigadores expertos en investigación con infantes, durante los años 2015 - 2017. Durante las Ilamadas telefónicas con los cuidadores, los investigadores tenían consigo una copia impresa del ChaT por participante, y anotaban en él las respuestas obtenidas. Así mismo, al finalizar las 10 preguntas, se recabaron los datos de la edad de ambos padres y se preguntó si había alguna duda sobre el estudio, brindando información pertinente en caso de que así fuera.

Las respuestas obtenidas en los cuestionarios contestados por los 325 cuidadores fueron capturadas en una base de datos diseñada expresamente para este estudio, identificando a cada participante con un número de 4 dígitos, la edad del infante y la fecha de aplicación. Una vez que se capturaron todas las participaciones, se realizaron análisis descriptivos y comparativos de cada una de las 10 preguntas del ChaT, los cuales se presentan en la siguiente sección. Todas las aplicaciones fueron archivadas en carpetas físicas y archivos digitales de manera confidencial.

\section{Resultados}

A continuación, se muestran los resultados de toda la muestra de infantes participantes (expresados en porcentajes), obtenidos en cada una de las preguntas que conforman el cuestionario. Dado que los cuidadores podían dar más de una respuesta en la mayoría de los reactivos, la suma de los porcentajes en cada pregunta no es igual al 100\%.

En relación a la Pregunta 1, se observó que más del 60\% tienen televisión, teléfono celular (inteligente y no inteligente), laptop, tableta electrónica y servicio de internet en su casa; y el medio favorito (Pregunta 2) por los infantes es la televisión, seguido del teléfono inteligente y la tableta electrónica.

Una de las variables de mayor interés del presente estudio era conocer la cantidad de tiempo que pasan los infantes con los dispositivos tecnológicos, diferenciando el consumo de acuerdo a cada dispositivo (Pregunta 3). En términos generales se observó que el dispositivo más utilizado es la televisión, llegando a consumir hasta más de 5 horas al día, seguido de la tableta electrónica y el servicio de internet con un máximo de cuatro 
horas al día. En la Tabla 1, se pueden observar los porcentajes correspondientes a las Preguntas 1 y 2 , y en la Figura 1 se ilustra el tiempo de consumo de los infantes con cada dispositivo expresado en porcentajes.

Tabla 1. Porcentajes correspondientes a los dispositivos que los infantes tienen en casa y el medio favorito de los infantes.

Tel Celular Laptop PC Internet Mp3 Video- Consola Tableta TV Intelijue go gente Portátil

\begin{tabular}{|l|l|l|l|l|l|l|l|l|l|l|}
\hline $\begin{array}{l}\text { Disposi- } \\
\text { tivos en } \\
\text { casa }\end{array}$ & $93.2 \%$ & $61.8 \%$ & $79.7 \%$ & $42.2 \%$ & $96.0 \%$ & $46.8 \%$ & $14.5 \%$ & $32.6 \%$ & $69.2 \%$ & $97.8 \%$ \\
\hline $\begin{array}{l}\text { M e d i o } \\
\text { Favorito }\end{array}$ & $44.0 \%$ & $6.2 \%$ & $2.8 \%$ & $4.3 \%$ & $4.0 \%$ & $0.3 \%$ & $0.6 \%$ & $1.8 \%$ & $33.2 \%$ & $50.5 \%$ \\
\hline
\end{tabular}

80

60

40

20

0
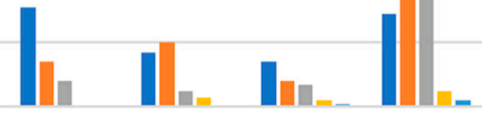

$c^{2 v^{2}}$ 
Respecto a la Pregunta 4 del cuestionario, se encontró que el $15.1 \%$ de los infantes sí tienen un dispositivo propio a comparación del $84.9 \%$ que no lo tienen. Los contenidos que más consumen los infantes (Pregunta 5) son en primer lugar videos y música por internet (79.1\%), seguido de programas de carácter infantil en servicio de televisión de paga (62.8\%) y en tercer lugar películas en formato DVD o internet (60.6\%). Otros contenidos como programas en televisión abierta, juegos, y contenido no infantil se consumen en menor porcentaje (39.4\%, 31.7\% y $24.3 \%$ respectivamente). En el caso del contenido no infantil, los cuidadores reportaron que sus hijos consumían ese contenido de forma indirecta, ya que en general los infantes están presentes con sus padres cuando éstos ven una película o serie de televisión.

En cuanto a la Pregunta 6, se encontró que el contexto en el que los infantes consumen más frecuentemente tecnología es en sus casas (96\%) o en casa de algún familiar (32.6\%). Otros contextos donde los infantes consumen tecnología son en el transporte (21.2\%), lugares públicos $(12.9 \%)$ y en menor medida la escuela (4\%). En la Pregunta 7, la mayoría de los participantes reportó que el consumo es bajo la supervisión de mamá o papá (72\%), aunque también utilizan los dispositivos de manera individual (49.5\%) o con hermanos (33.2\%) y otros adultos (26.8\%).

Como se observó anteriormente, el CHaT contempla el tiempo que los infantes dedican a las actividades de juego y lectura sin considerar los dispositivos tecnológicos. En estas preguntas (8 y 9) se encontró que el 36\% de los infantes pasan más de cinco horas al día en actividad de juego, y que el $44 \%$ dedica menos de dos horas al día en actividades de lectura.

Finalmente, en la Pregunta 10, se observó que la mayoría de los participantes (69.2\%) considera el uso de dispositivos tecnológicos como una actividad positiva para los infantes.

\section{Efecto de la edad}

Al analizar los datos por rangos, se encontró que el consumo tecnológico de los infantes de los diferentes grupos de edad presenta la misma tendencia que el promedio global, en la 
mayoría de los reactivos del cuestionario. Por ejemplo, se observó que el medio favorito de los infantes en todos los grupos de edad es la televisión, seguida del teléfono inteligente y la tableta electrónica. Del mismo modo que en el promedio global, el reproductor de mp3 y los videojuegos, representan menos del $2 \%$ de consumo en todas las edades estudiadas. Sin embargo, el porcentaje de infantes que prefiere cierto dispositivo (Pregunta 2), por ejemplo la televisión, aumenta conforme aumenta la edad de los mismos. Este hallazgo puede ser útil como indicador de consumo, infiriendo que los infantes de mayor edad son más propensos a consumir contenidos en más de un dispositivo tecnológico en un mismo día.

También, se observaron diferencias entre los grupos de edad en otras dos de las diez preguntas del cuestionario. Una de ellas se refiere a la Pregunta 4 ¿El infante tiene dispositivo propio?, pues dicho porcentaje aumentó conforme a la edad de los infantes de $6.7 \%$ hasta $33.33 \%$ (12-23 meses $=6.7 \% ; 24-29$ meses $=12.9 \%$; 30-35 meses $=16.41 \%$; 36-41 meses $=16.66 \%$; $42-47$ meses $=16 \%$; y 48 a 72 meses $=33.33 \%$ ). Cabe resaltar que la mayor diferencia se observó a partir de los 4 años de edad, en la que el porcentaje de infantes con dispositivo propio casi se duplica, respecto a los porcentajes de los rangos de edad anteriores.

Respecto a la Pregunta 10 ¿Cómo considera el uso de dispositivos con su bebé?, se observó un efecto de la edad sobre la valoración que hacen los cuidadores, sobre si el uso de dispositivos por parte de los infantes es positivo o negativo. Los resultados en este estudio mostraron que conforme aumenta la edad de los niños, también aumenta la percepción positiva de los padres sobre el uso de los dispositivos. Específicamente se encontró que para los infantes más pequeños (12-23 meses) el 59.32\% de los padres considera positivo el uso de dispositivos; en el segundo rango (24-29 meses) se observó un $61.29 \%$ de aceptación; en el tercer rango (30-35 meses) un 62.68\%; en el cuarto rango de edad (36-41 meses) se observó un 70\%; y en el quinto (42-47 meses) y sexto rango de edad (48-72 meses) se observó un porcentaje de aceptación del $76 \%$ y $77.77 \%$ respectivamente. 


\section{Discusión}

Los resultados obtenidos en esta investigación son congruentes con los hallazgos mostrados en estudios previos (Gutnick et al., 2011; Radesky et al., 2015) respecto al tipo de dispositivos que prefieren los infantes, así como al tiempo de consumo. Específicamente, se encontró que tanto infantes como preescolares tienen un medio favorito bien definido, siendo éste la televisión por encima de los demás dispositivos tecnológicos. De acuerdo a Radesky (2015), la televisión ha tenido un impacto claramente documentado, tanto positivo como negativo, en los niños. Los dispositivos móviles, debido a su portabilidad y componentes interactivos, están introduciendo los medios en todos los aspectos de la experiencia de los niños y merecen una seria atención e investigación. Es un dato de interés el hecho que a pesar de la fácil accesibilidad que tienen los infantes hacia los dispositivos móviles, prefieren consumir contenido en la televisión.

Este último dato se complementa con la información sobre el tiempo que los infantes pasan consumiendo tecnología. En este estudio se observó que la mayoría de los niños participantes (87\%) ven televisión menos de dos horas al día. Este porcentaje es mayor en el caso del uso de la tableta electrónica y el celular inteligente, siendo el $97.9 \%$ y el $99.1 \%$ de infantes respectivamente que consumen dichos dispositivos menos de dos diarias. No obstante, hay que tener presente que sí hay un pequeño porcentaje de infantes que sobrepasan las dos horas diarias de consumo de estos dispositivos. En el caso de la televisión el 1.5\% de los infantes llega a consumir hasta 5 horas al día; y con la tableta electrónica el .3\% de los infantes pasa más de dos horas al día con dicho dispositivo. De acuerdo a la literatura previa (Radesky et al., 2015; Séguin \& Klimek, 2016), sobrepasar dos horas diarias de consumo televisivo en edades tempranas, puede derivar en problemas de conducta y en hábitos de higiene como la cantidad de horas de sueño, a corto y largo plazo. Si bien es bajo el porcentaje de infantes que reportan alto consumo tecnológico, es necesario monitorear su conducta 
y la dinámica familiar para que dicho porcentaje no vaya en aumento y de esta forma evitar problemas de salud y conductuales más adelante en el desarrollo.

Vigilar el uso de la tecnología por los infantes puede resultar más efectivo cuando no se les proporciona un dispositivo propio, al menos en edades muy tempranas. En este estudio se encontró que el $15.1 \%$ de los infantes poseen un dispositivo propio y que dicho porcentaje aumenta conforme a la edad de los infantes hasta un 33\%. Este último hallazgo fue diferente respecto a estudios previos que han reportado hasta un $75 \%$ de infantes con dispositivo propio, en el mismo rango de edad (Kabali et al., 2015)low-income, minority community. The survey was adapted from Common Sense Mediass 2013 nationwide survey. RESULTS Most households had television (97\%. Sin embargo, la tendencia con niños mexicanos es que la cantidad de infantes con dispositivo propio va en aumento, lo cual también merece atención desde la investigación y las prácticas parentales, para evitar efectos negativos en la salud de los niños.

Por otro lado, los contenidos reportados en este estudio son en su mayoría videos y música a través de internet (hasta $47 \%$ ), y los juegos sólo representan entre 10 y 25\% en todos los rangos de edad. Por el contrario, la actividad de juego sin dispositivos tecnológicos llega a ser más de 5 horas al día en el 36\% de los infantes, hecho que favorece el desarrollo motriz y cognitivo de los infantes a estas edades. Respecto a la actividad de lectura por parte de los infantes, se encontró que el 44\% sí realiza esta actividad menos de dos horas al día. Se considera deseable que los infantes tengan contacto temprano con material de lectura, pues favorece el desarrollo del lenguaje y vocabulario entre otras habilidades. En este aspecto se pueden implementar los libros electrónicos (e-books), ya que es una manera de fomentar la actividad intelectual infantil, siempre y cuando sea a través del uso supervisado y dirigido por los cuidadores (Kucirkova \& Zuckerman, 2017; Rees et al., 2017; Strouse \& Ganea, 2017; Troseth \& Strouse, 2017)specifically comparing children with typically developing language (TD.

En los resultados de este estudio también se observó que el juicio positivo que emiten los cuidadores sobre el uso de dis- 
positivos depende de la edad de los niños. De acuerdo a los resultados reportados en estudios previos y el presente, la edad de 4 años resulta crítica en los hábitos de consumo de tecnología, específicamente de dispositivos móviles. Es a esta edad en la que se ha observado un aumento de dispositivos propios, más consumo simultáneo de dispositivos y una mayor aceptación por parte de los cuidadores hacia la manipulación tecnológica. Lo anterior puede deberse a que alrededor de esta edad, los infantes comienzan su educación preescolar y la dinámica cotidiana cambia constantemente. Al estar los infantes en un sistema escolarizado, los cuidadores los perciben "más grandes e independientes" flexibilizando el tipo de dispositivos que utilizan, así como el tiempo de consumo.

El presente estudio aporta datos relevantes sobre los hábitos tecnológicos de niños desde edades tempranas, extiende dichos hallazgos a un mayor rango de edad con una mayor muestra, en comparación con la literatura previa en población mexicana. Este trabajo se ofrece como una herramienta de consulta sobre la conducta de infantes y preescolares hispanohablantes respecto al consumo tecnológico; así como un recurso útil para la toma de decisiones informada sobre la pertinencia del uso de dispositivos móviles en edades tempranas. Se requiere más investigación para realizar recomendaciones informadas y basadas en evidencia, tanto para los profesionales de la salud como para las familias sobre el uso de medios móviles por parte de niños pequeños.

\section{Referencias}

Gutnick, A. L., Robb, M., Takeuchi, L., \& Kotler, J. (2011). Always connected. Sesame Workshop, 21. https://doi.org/www. joanganzcooneycenter.org.

Kabali, H. K., Irigoyen, M. M., Nunez-Davis, R., Budacki, J. G., Mohanty, S. H., Leister, K. P., \& Bonner, R. L. (2015). Exposure and Use of Mobile Media Devices by Young Children. Pediatrics, 136(6), 1044-1050. https://doi.org/10.1542/ peds.2015-2151

Karemaker, A., Jelley, F., Clancy, C., \& Sylva, K. (2017). The 
effects on children's literacy skills of reading e-books with different features: Are "bells and whistles" over-rated? International Journal of Child-Computer Interaction, 12, 3036. https://doi.org/10.1016/j.ijcci.2017.01.004

Kucirkova, N., \& Zuckerman, B. (2017). A guiding framework for considering touchscreens in children under two. International Journal of Child-Computer Interaction, 12, 46-49. https://doi.org/10.1016/j.ijcci.2017.03.001

McClure, E., Vaala, S., \& Spiewak Toub, T. (2017). Discovering kids' apps: do family strategies vary by income? Sesame Workshop, 1-14. Retrieved from http://joanganzcooneycenter.org/wp-content/uploads/2017/06/jgcc_discoveringkidsapps.pdf\%0AThe Joan Ganz Cooney Center at Sesame Workshop

McDaniel, B. T., \& Radesky, J. S. (2018). Technoference: Parent Distraction With Technology and Associations With Child Behavior Problems. Child Development, 89(1), 100-109. https://doi.org/10.1111/cdev.12822

McManis, L. D., \& Gunnewig, S. B. (2012). Finding the Education in Educational Technology with Early Learners. Young Children, 67(May), 14-24.

Radesky, J. S., Schumacher, J., \& Zuckerman, B. (2015). Mobile and Interactive Media Use by Young Children: The Good, the Bad, and the Unknown. Pediatrics, 135(1), 1-3. https:// doi.org/10.1542/peds.2014-2251

Rees, K., Nadig, A., \& Rvachew, S. (2017). Story-related discourse by parent-child dyads: A comparison of typically developing children and children with language impairments. International Journal of Child-Computer Interaction, 12, 16-23. https://doi.org/10.1016/j.ijcci.2017.01.001

Rvachew, S., Rees, K., Carolan, E., \& Nadig, A. (2017). Improving emergent literacy with school-based shared reading: Paper versus ebooks. International Journal of Child-Computer Interaction, 12, 24-29. https://doi.org/10.1016/j.ijcci.2017.01.002

Séguin, D., \& Klimek, V. (2016). Just five more minutes please: electronic media use, sleep and behaviour in young children. Early Child Development and Care, 186(6), 981- 
1000. https://doi.org/10.1080/03004430.2015.1071528 Slutsky, R., \& DeShetler, L. M. (2017). How technology is transforming the ways in which children play. Early Child Development and Care, 187(7), 1138-1146. https://doi.org/10.1 080/03004430.2016.1157790

Strouse, G. A., \& Ganea, P. A. (2017). A print book preference: Caregivers report higher child enjoyment and more adult-child interactions when reading print than electronic books. International Journal of Child-Computer Interaction, 12, 8-15. https://doi.org/10.1016/j.ijcci.2017.02.001

Suárez, P., Alva, E., \& Ferreira, E. (2016). Technological habits in Mexican children younger than 4 years of age. In SRCD Topic Meeting. Technology and media in children. Irvine, California.

Suárez, P., Ferreira, E., \& Alva, E. (2016). Hábitos tecnológicos en infantes mexicanos. In III Congreso Internacional de Innovación Educativa (CIIE). Ciudad de México.

Suárez, P., Perez, K., Ferreira, E., \& Alva, E. A. (2016). Uso de dispositivos tecnológicos en infantes menores de 4 años. In Congreso Internacional de Psicología del Desarrollo. Aguascalientes, Aguascalientes.

Troseth, G. L., \& Strouse, G. A. (2017). Designing and using digital books for learning: The informative case of young children and video. International Journal of Child-Computer Interaction, 12, 3-7. https://doi.org/10.1016/j.ijcci.2016.12.002

Yienger, M. E. (2016). Too Much Tech Harms Reading Retention in Young Children. Inquiries Journal/Student Pulse, 8(3), $1-4$.

Zosh, J. M., Hirsh-Pasek, K., Golinkoff, R. M., \& Parish-Morris, J. (2016). Learning in the Digital Age: Putting Education Back in Educational Apps for Young Children, 1-5. 